\title{
Acid ceramidase confers radioresistance to glioblastoma cells
}

\author{
NINH B. DOAN ${ }^{1,2}$, HA S. NGUYEN ${ }^{2}$, MONA M. AL-GIZAWIY ${ }^{3}$, WADE M. MUELLER ${ }^{2}$, \\ ROGER A. SABBADINI ${ }^{9}$, SCOTT D. RAND ${ }^{3}$, JENNIFER M. CONNELLY ${ }^{4}$, \\ CHRISTOPHER R. CHITAMBAR ${ }^{5}$, KATHLEEN M. SCHMAINDA ${ }^{3,6}$ and SHAMA P. MIRZA ${ }^{1,7,8}$ \\ ${ }^{1}$ Biotechnology and Bioengineering Center, Departments of ${ }^{2}$ Neurosurgery, ${ }^{3}$ Radiology, ${ }^{4}$ Neurology, ${ }^{5}$ Medicine, \\ Hematology/Oncology, ${ }^{6}$ Biophysics and ${ }^{7}$ Obstetrics and Gynecology, Medical College of Wisconsin, Milwaukee, \\ WI 53226; ${ }^{8}$ Department of Chemistry and Biochemistry, University of Wisconsin-Milwaukee, Milwaukee, WI 53211; \\ ${ }^{9}$ Department of Biology, San Diego State University, and Lpath Inc., San Diego, CA 92121, USA
}

Received January 2, 2017; Accepted June 19, 2017

DOI: $10.3892 /$ or.2017.5855

\begin{abstract}
Glioblastoma multiforme (GBM) is the most common primary, intracranial malignancy of the central nervous system. The standard treatment protocol, which involves surgical resection, and concurrent radiation with adjuvant temozolomide (TMZ), still imparts a grim prognosis. Ultimately, all GBMs exhibit recurrence or progression, developing resistance to standard treatment. This study demonstrates that GBMs acquire resistance to radiation via upregulation of acid ceramidase (ASAH1) and sphingosine-1-phosphate (Sph-1P). Moreover, inhibition of ASAH1 and Sph-1P, either with humanized monoclonal antibodies, small molecule drugs (i.e. carmofur), or a combination of both, led to suppression of GBM cell growth. These results suggest that ASAH1 and Sph-1P may be excellent targets for the treatment of new GBMs and recurrent GBMs, especially since the latter overexpresses ASAH1.
\end{abstract}

\section{Introduction}

Acid ceramidase (ASAH1), a lysosomal cysteine amidase, helps metabolize ceramides into sphingosine and free fatty acids. Ceramides promote senescence and apoptosis, while sphingososine-1-phospate (Sph-1P), the immediate metabolite of sphingosine, promotes cell survival, proliferation, inflammation, and angiogenesis (1). As such, overexpression of ASAH1 confers resistance to apoptosis. Its levels have been shown to be elevated in several cancers, including breast (2), prostate $(3,4)$, head and neck $(5)$, colon, and melanoma (6).

Correspondence to: Dr Ninh Doan, Biotechnology and Bioengineering Center, Medical College of Wisconsin, Milwaukee, WI 53226, USA

E-mail: ndoan@mcw.edu

Key words: glioblastoma, acid ceramidase, acid ceramidase inhibitors, carmofur, radioresistance, radiation, sphingosine, sphingosine-1-phosphate, Sph-1P, deoxyribonucleic acid, DNA
Moreover, downregulation or inhibition of ASAH1 may improve anticancer treatments $(5,7,8)$.

Glioblastoma multiforme (GBM) is the most common primary, intracranial malignancy of the central nervous system. The standard treatment protocol, which involves surgical resection, concurrent radiation/temozolomide (TMZ), and adjuvant $\mathrm{TMZ}$, still imparts a grim prognosis, where median overall survival (OS) is less than 15 months (9). Ultimately, all GBM develop resistance to standard treatment with recurrence or progression, where additional therapies yield a median survival of $\sim 30$ weeks (10-12). Though ASAH1 appears to be a promising therapeutic target in other tumors, no studies have explored its role in recurrent GBM.

It has been postulated that GBM cancer stem cells (CSCs) are the culprits that promote resistance to radiation, as CD133carrying glioma cells are increased in proportion following ionizing radiation (13). Recent studies with prostate cancer suggest that upregulation of ASAH1 confers resistance to radiation by altering the sphingolipid metabolism pathway $(14,15)$. This study examines whether ASAH1 plays a similar role in recurrent or irradiated GBM.

\section{Materials and methods}

Reagents and cells. Mouse antibody against ASAH1 (612302) was purchased from BD Biosciences (San Jose, CA, USA). Anti-actin, carmofur, temozolomide (TMZ), and $N$-oleoylethanolamine (OE), 3-(4,5-dimethylthiazol-2-yl)2,5-diphenyltetrazolium bromide (MTT) were purchased from Sigma-Aldrich (St. Louis, MO, USA). HRP-conjugated goat anti-mouse IgG was supplied by R\&D Systems, Inc. (Minneapolis, MN, USA). SDS-PAGE and western blot materials were obtained from Life Technologies, Inc. (Grand Island, NY, USA). Murine anti-Sph-1P monoclonal antibody, (LT1002) and humanized anti-Sph-1P monoclonal antibody (LT1009) were obtained from Lpath, Inc. $(16,17)$.

Cells. The pediatric glioblastoma cell line (SJGBM2) was obtained from the Children's Oncology Group (COG) Cell Culture and Xenograft Repository. These cells were grown in Iscove's modified Dulbecco's medium supplemented with $20 \%$ 
fetal bovine serum, $4 \mathrm{mM} \mathrm{L}$-glutamine, and 1X ITS ( $5 \mu \mathrm{g} / \mathrm{ml}$ insulin, $5 \mu \mathrm{g} / \mathrm{ml}$ transferrin, $5 \mathrm{ng} / \mathrm{ml}$ selenous acid). The U87 glioblastoma cell line was cultured in Eagle's minimum essential medium (MEM) containing 10\% (v/v) fetal bovine serum (FBS).

Radiation. Cells (U87 and SJGBM2) were grown to confluence, and then radiated with a Pantak HF320 X-ray machine (Agfa NDT Ltd., Reading, UK) operating at $300 \mathrm{kV}$ at a dosage of $2.09 \mathrm{~Gy} / \mathrm{min}$ to a total radiation dose of $10 \mathrm{~Gy}$, to generate the U87-10gy and SJGBM2-10gy cell lines. Following radiation, these irradiated cells were allowed to grow to confluence over a period of $\sim 1$ month prior to any experiments that were performed.

Tissue collection. All human brain samples were collected after informed written consent was obtained from the GBM patients. The research protocol was approved by the Institutional Review Board (IRB) at the Medical College of Wisconsin (MCW), Milwaukee, WI, USA. Briefly, glioblastoma tumor tissues from consented patients were collected at the time of tissue resection and snap-frozen in liquid nitrogen within 30 min of removal and stored at $-80^{\circ} \mathrm{C}$ in the Brain and Spinal Tissue Bank at MCW until use. All tissues were evaluated by routine histologic, immunohistochemical, and angiogenic measurements. Each tissue biopsy sample was fixed in $10 \%$ buffered formalin, processed, embedded in paraffin, cut, stained with hematoxylin and eosin and any other histochemical or immunohistochemical stains needed to fully evaluate the tissue. The diagnostic evaluation of each biopsy was performed in the Department of Pathology at MCW. Diagnosis of glioblastoma was based on morphologic features that are considered histological hallmarks of glioblastoma, including high cellularity, nuclear hyperchromatism and pleomorphism, abundant mitoses, endothelial proliferation, and necrosis with or without pseudo-palisades per the WHO classification.

Tissue homogenization. GBM primary tumor samples were homogenized and powdered in liquid nitrogen using a mixer mill (Retsch Inc., Haan, Germany). Samples were maintained at liquid $\mathrm{N} 2$ temperature throughout the process. Homogenized and powdered tissue samples were then re-suspended in $5 \mathrm{X}$ volume of the weight of the tissue sample in a reducing buffer (125 mM Tris pH 6.8, 4\% SDS (w/v), 10\% glycerol (v/v), 5\% 2-mercaptoethanol (v/v), complete protease inhibitor (Roche Diagnostics Corp., Indianapolis, IN, USA), HALT phosphatase inhibitor (Thermo Fisher Scientific, Grand Island, NY, USA). Samples were then heated to $70^{\circ} \mathrm{C}$ with mixing at $1,400 \mathrm{rpm}$ for $10 \mathrm{~min}$, sonicated with a tip sonicator for $30 \mathrm{sec}$ at power level 4 , and then centrifuged at $16,000 \mathrm{x}$ g for $10 \mathrm{~min}$ at room temperature. The supernatant was then collected.

Western blot analysis and quantification. Equal amounts $(15 \mu \mathrm{g})$ of protein from each of the tumor samples were loaded onto the $4-12 \%$ gel. SDS-PAGE and western blots were performed using standard methods. Gels were blocked with 5\% bovine serum albumin. A 1:500 dilution was used for primary antibody and 1:10,000 for secondary antibody. ImageJ software was used to quantify western blot images.
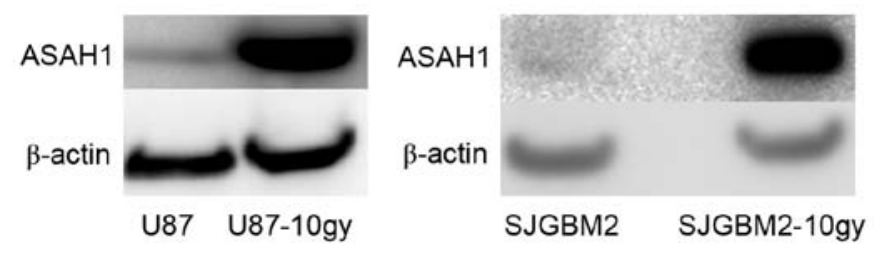

Figure 1. U87-10gy and SJGBM2-10gy cells overexpressed ASAH1. U87 and SJGBM2 were irradiated (10 Gy) to produce U87-10gy and SJGBM2-10gy, respectively. Irradiated cells were allowed to growth to confluence prior to assaying. Protein lysates were subjected to western blot analysis for ASAH1 protein expression. Results shown are representatives of three independent experiments.

Acid ceramidase immunohistochemistry (IHC) methodology. All immunohistochemical (IHC) staining was performed on a Dako Autostainer Plus using the Dako Envision ${ }^{\mathrm{TM}}$ FLEX High pH detection kit. Briefly, after deparaffinization and rehydration of the tissue, antigen retrieval was performed with Tris/EDTA pH 9. After blocking of non-target epitopes, antiacid ceramidase primary antibody (Santa Cruz Biotechnology Inc., Dallas, TX, USA) was applied at a concentration of 1:100 for $30 \mathrm{~min}$, secondary antibody for $20 \mathrm{~min}$, and DAB for $10 \mathrm{~min}$. Hematoxylin was used as counterstain.

Immunohistochemistry (IHC) scoring. Photomicrographs of stained tissues were acquired and graded blindly using the Allred scoring system as follows. For each patient, we determined the proportion of positively stained tumor cells (proportion score 'PS'), as well as the staining intensity (mean intensity score 'IS') (18). Both scores were added together to obtain the final Allred score, which was then matched with the individual WHO pathology diagnoses.

Sphingolipid quantification. Electrospray ionization tandem mass spectrometry (ESI/MS/MS) analysis of endogenous (phyto)ceramide species were performed on a Thermo Fisher Quantum triple quadrupole mass spectrometer, operating in a multiple reaction monitoring (MRM) positive ionization mode, using the modified version of published methods (19). Briefly, biological materials were fortified with the internal standards (ISs: $\mathrm{C}_{17}$ base D-erythro-sphingosine (17CSph), $C_{17}$ sphingosine-1-phosphate (17CSph-1P), $\mathrm{N}$-palmitoyl-D-erythro- $\mathrm{C}_{13}$ sphingosine $(13 \mathrm{C} 16-\mathrm{Cer})$ and heptadecanoyl-D-erythro-sphingosine (C17-Cer) and C6-Phyto-ceramide), then extracted with an ethyl acetate/ iso-propanol/water $(60 / 30 / 10 \% \mathrm{v} / \mathrm{v})$ solvent system. After evaporation and reconstitution in $150 \mu 1$ of methanol, samples were injected on the HP1100/TSQ Quantum LC/MS system and gradient eluted from the BDS Hypersil C8, 150x3.2 mm, $3 \mu \mathrm{m}$ particle size column, with a $1.0 \mathrm{mM}$ methanolic ammonium formate $/ 2 \mathrm{mM}$ aqueous ammonium formate mobile phase system. Peaks corresponding to the target analytes and internal standards were collected and processed using the Xcalibur software system. Quantitative analysis was based on the calibration curves generated by spiking an artificial matrix with the known amounts of the target analyte synthetic standards and an equal amount of the internal standards (ISs). The target analyte/IS peak area ratios were plotted against analyte concentration. The target analyte/IS 
A

U87

U87-10gy

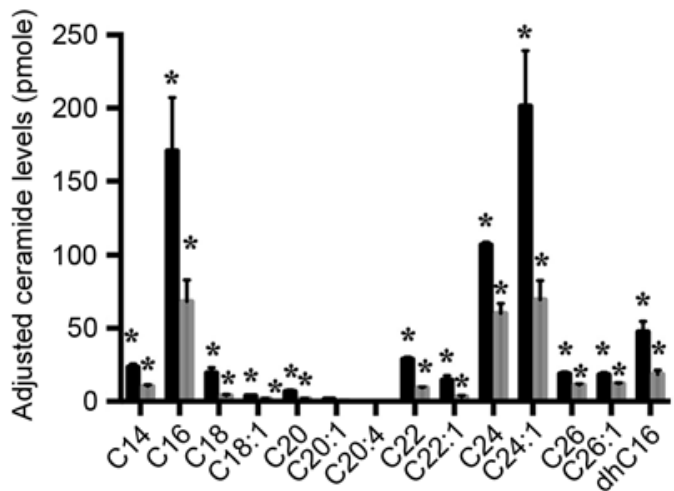

B

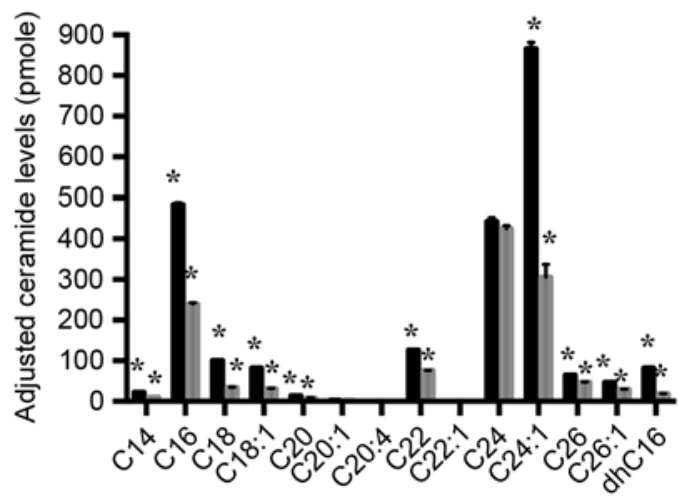

Figure 2. Ceramides are downregulated in both irradiated U87 and SJGBM2 cells (U87-10gy and SJGBM2-10gy, respectively). Cell pellets were prepared and lipids were extracted for mass spectrometry as described in Materials and methods. (A) Intracellular ceramide levels from U87 (black bars) and U87-10gy (gray bars) cells. (B) Intracellular ceramide levels from SJGBM2 (black bars) and SJGBM2-10gy (gray bars) cells. Concentrations were normalized and adjusted based on the phosphate level. $\mathrm{C}(\mathrm{N})$, ceramide species with long-N-acyl-chain; dhC, dihydroceramides. Results shown are mean $\pm \mathrm{SD}$ of three independent experiments. ${ }^{*} \mathrm{P}<0.05$.

A B

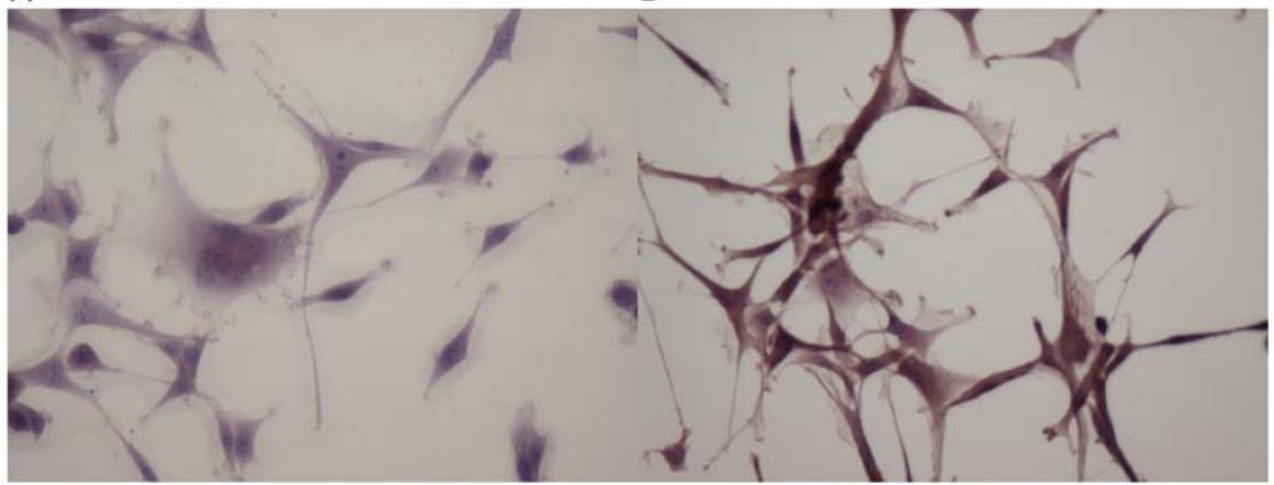

Figure 3. Sphingosine-1-phosphate is upregulated following radiation. Cells were grown on a slide and then fixed for IHC. Using the murine anti-Sph-1P antibody (LT1009), IHC was performed on (A) U87 and (B) U87-10gy cells. Comparing to U87 cells, much greater staining intensity (reddish color) was detected in U87-10gy cells. Results shown are representatives of three independent experiments.

peak area ratios from the samples were similarly normalized to their respective ISs and compared to the calibration curves, using a linear regression model. Introduction of the internal standards to the samples prior to extraction, yielded results already 'recovery corrected', therefore, no further data manipulation was necessary.

\section{3-(4,5-Dimethylthiazol-2-yl)-2,5-diphenyltetrazolium bromide} (MTT) assays. Cells were plated onto a 96-well plate at the density of $1 \times 10^{5}$ cells $/ \mathrm{ml}$. Media was exchanged to serum-free media following overnight incubation. Cells were treated with various antibodies for $48 \mathrm{~h}$. MTT reagents were added after $48 \mathrm{~h}$ of incubation, followed by acidic-isopropanol $4 \mathrm{~h}$ later to dissolve formazan. The absorbance values were recorded at wavelengths 570 and $630 \mathrm{~nm}$. $\mathrm{IC}_{50}$ values were calculated with the GraphPad Prism software.

Immunohistochemistry (IHC) of Sph-1P. Cells were grown on Nunc Lab-Tek Chamber slides overnight then fixed and stained according to the published protocol, with the exception that the staining of U87 and U87-10gy cells was performed with LT1002 at the concentration of $22 \mu \mathrm{g} / \mathrm{ml}(16,20)$. Slides were imaged with a Nikon Eclipse 80i microscope at the mentioned magnification level.

\section{Results}

U87-10gy and SJGBM2-10gy cells overexpress ASAH1. A previous study demonstrated the upregulation of ASAH1 in irradiated prostate cancer cells, suggesting a mechanism of cancer cell resistance to radiation (14). To evaluate the role of ASAH1 in promoting radioresistance, native U87 and SJGBM2 cells were irradiated with 10 Gy of radiation to generate U87-10gy and SJGBM2-10gy cell lines. Less than $1 \%$ of total cells survived radiation. Irradiated cells were allowed to grow to confluence prior to being harvested for assays. Western blots of the cells demonstrated U87-10gy and SJGM2-10gy cell lines expressed much higher levels of ASAH1 compared to their native counterparts (Fig. 1). These findings indicate that these survived cells naturally 
A
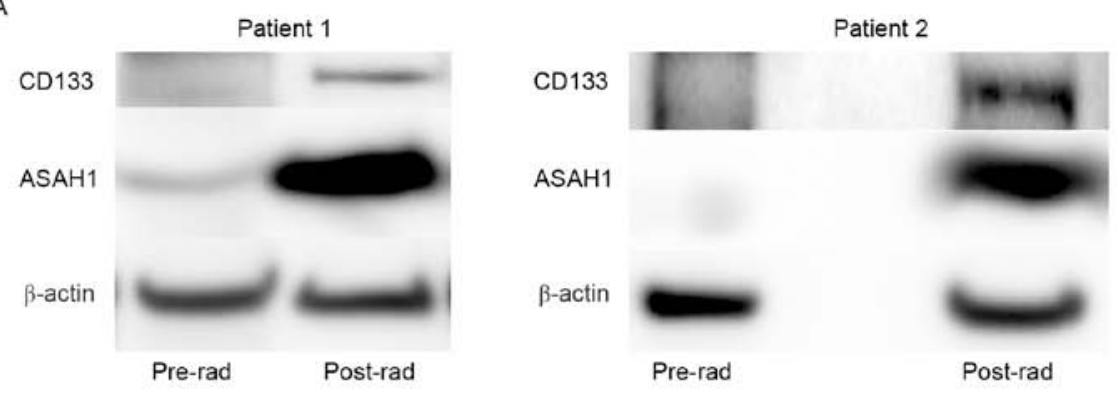

B
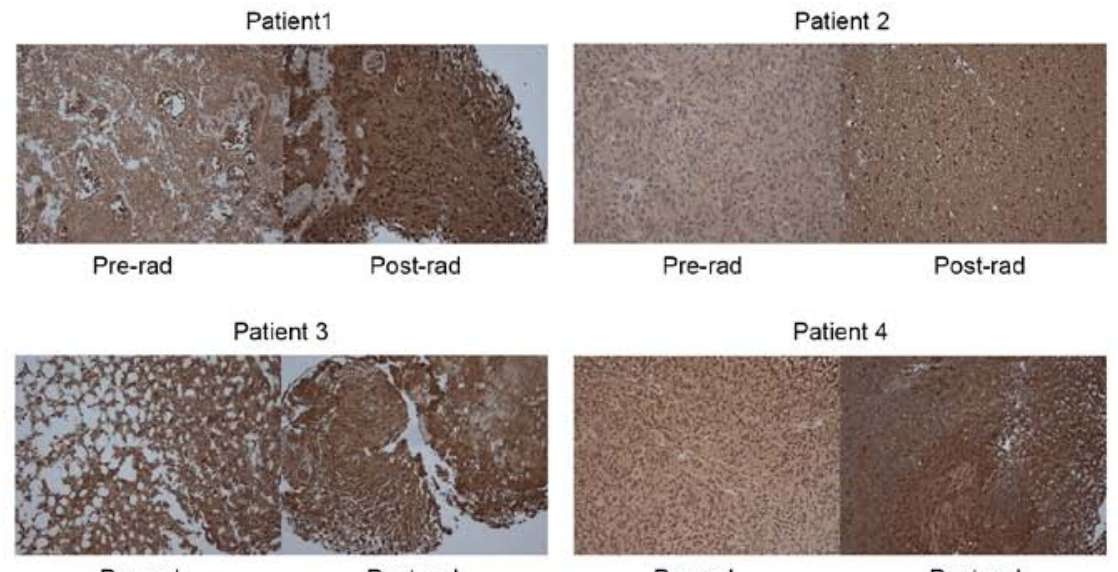

Pre-rad

Post-rad

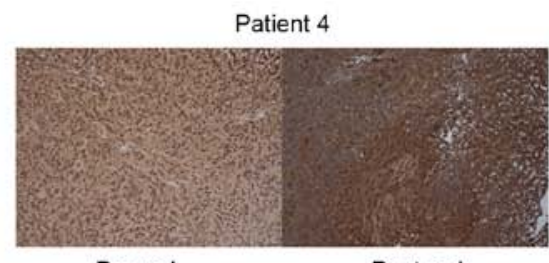

Pre-rad

Post-rad

C
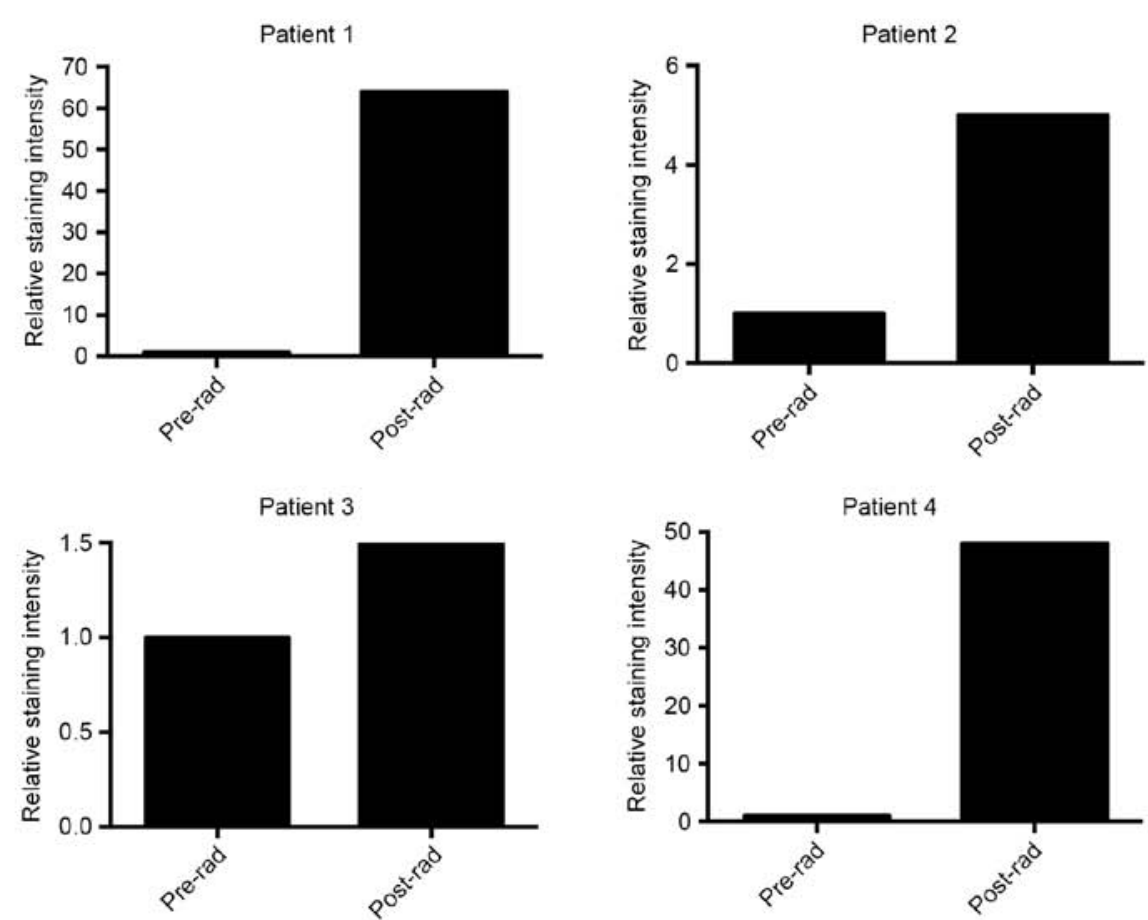

Figure 4. ASAH1 and CD133 are upregulated in irradiated or recurrent patient GBM tissues. Pre-radiation (Pre-rad) and post-radiation (Post-rad) GBM tissues from the same patients who had undergone radiotherapies and yet still having recurrent GBMs were homogenized and subjected to western blotting and IHC with anti-ASAH1 and anti-CD133 antibodies. (A) Protein lysates were subjected to western blot analysis for ASAH1 and CD133 protein expression. Results shown are representatives of three independent experiments (B) IHC of GBM tissues with the anti-ASAH1 antibody, images were taken with a magnification of x10. (C) ASAH1 staining intensities were quantified with ImageJ and displayed as relative intensity with respect to the pre-radiation tissues.

overexpress ASAH1, which may be an important mechanism for cell survival after radiation.

Cell radiation results in reduced accumulation of ceramides in both U87-10gy and SJGBM2-10gy. To determine whether upregulation of ASAH1 modulates sphingolipid metabolism, sphingolipid levels were determined in native and irradiated GBM cells. In addition to the accumulation of ASAH1, cells that survived radiation contained substantially reduced levels of all ceramides measured compared to native cells, potentially 
A

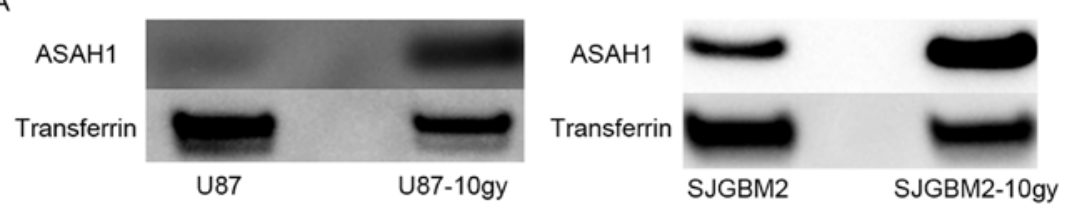

B

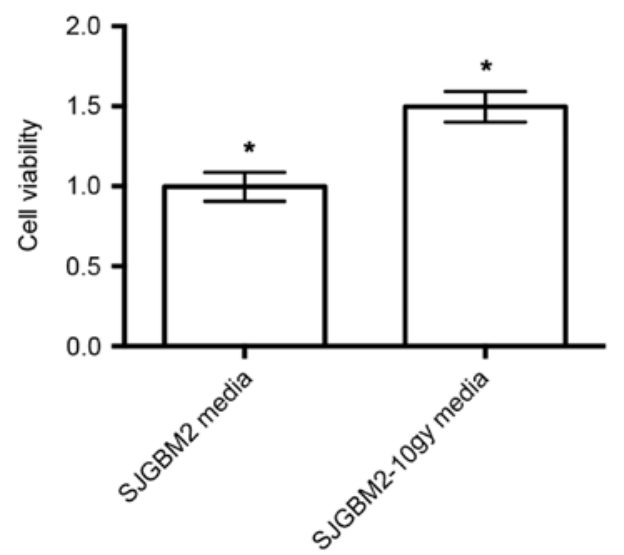

Figure 5. Radiation induced over-secretion of ASAH1 and serum-free media rich in secreted ASAH1 stimulated 50\% more cell growth. (A) SJGBM2 cells were irradiated $(10 \mathrm{~Gy})$ to produce U87-10gy and SJGBM2-10gy cells. Irradiated cells were allowed to growth to confluence prior to assaying. Cells were grown in serum-free media $\mathrm{x} 48 \mathrm{~h}$ and media of respective cell lines were then subjected to western blot analysis for secreted ASAH1 protein levels. (B) SJGM2 and SJGBM2-10gy were grown in serum-free media for $48 \mathrm{~h}$. These media were harvested and used to cultivate U87 cells for another $48 \mathrm{~h}$. Following this, cell growth of U87 cells cultivated with SJGBM2 media and SJGBM2-10gy media was calculated with MTT assays. Results shown are mean \pm SD of three independent experiments. ${ }^{*} \mathrm{P}<0.05$.

making them much less susceptible to cell death or apoptosis induced by chemo- or radiotherapy (Fig. 2).

Sphingosine-1-phosphate is upregulated following radiation as detected by IHC. To study the effect of radiation on the Sph-1P level, we elected to perform IHC, using the anti-Sph-1P murine monoclonal antibody to detect Sph-1P levels in U87 and U87-10gy cells. As shown in Fig. 3, U87-10gy cells exhibited a much greater staining intensity than U87 cells, suggesting the presence of a higher amount of Sph-1P in U87-10gy cells. A plausible interpretation is that radio-resistant cells contain a substantially elevated level of ASAH1, whose enzyme activity is known to be involved in the pathway leading to the accumulation of Sph-1P (as seen in Fig. 3) (4).

Irradiated or recurrent patient GBM tissues exhibited upregulation of ASAHI and CDI33 based on western blotting and $I H C$. We have now shown that irradiated cell culture tissues (U87-10gy and SJGBM2-10gy) have higher expression levels of ASAH1 than non-irradiated culture tissues. However, whether similar findings can be seen in native human GBM samples that had undergone prior radiation remains to be answered. To address this question, we obtained pre- and post-radiation GBM samples from the same patients who had undergone radiotherapies and eventually were diagnosed with recurrent GBM. Western blots of these homogenized patient tissues also demonstrated upregulation, to various degrees, of ASAH1 in post-radiation tissues in comparison to pre-radiation tissues, which paralleled the findings in tissue culture studies (Figs. 1 and 4A). CD133, a glioma cancer stem cell marker, was also found to be upregulated in post-radiation tissues (Fig. 4A). Similarly, IHC of these GBM samples revealed far greater ASAH1 staining in post-radiation samples when compared to pre-radiation samples (Fig. 4B and C). Postradiation GBMs also demonstrated higher ASAH1 staining in the background or extracellular space and this could be due to the secretion of ASAH1 into the extracellular space by irradiated GBMs, as had been shown to be the case in irradiated U87 and SJGBM2 cells (Fig. 5).

Radiation induces over-secretion of ASAHI and SJGBM210gy media containing a high amount of secreted ASAHI stimulated 50\% more cell growth than SJGBM2 media with a lower amount of secreted ASAH1. Western blots of serum-free media previously used to culture U87-10gy and SJGBM2-10gy demonstrated that U87-10gy and SJGM2-10gy cell lines secreted much higher levels of ASAH1 compared to their native counterparts (Fig. 5A). Far more ASAH1 was detected in the serum-free media of SJGBM2-10gy cells than from SJGBM2. To test the effect of ASAH1 on cell growth, serum-free media that were used to cultivate SJGBM2 and SJGBM2-10gy cells over a period of $48 \mathrm{~h}$ were collected. Irradiated SJGBM2-10gy cells were allowed to grow to confluence over a period of a week prior to being cultured for another $48 \mathrm{~h}$ in serum-free media. U87 cells were then grown in these serum-free media from irradiated SJGBM2$10 \mathrm{gy}$ cells for $48 \mathrm{~h}$ following by cell growth analysis with MTT assays. Consistent with its function, SJGBM2-10y media, which is rich in secreted ASAH1, promoted 50\% more cell growth than SJGBM2 media containing a lower level of secreted ASAH1 (Fig. 5B).

Neutralization of secreted ASAHI and Sph-1P with antiASAH1 and anti-Sph-1P antibodies, respectively, resulted 
A

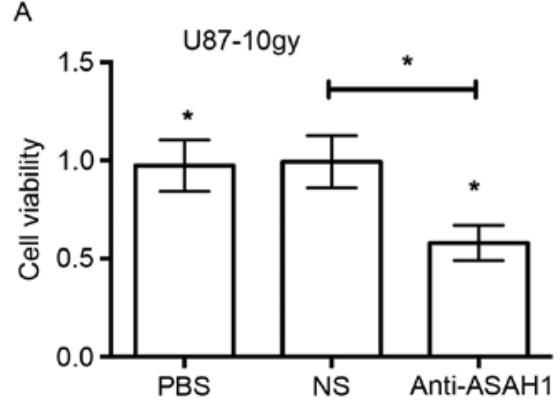

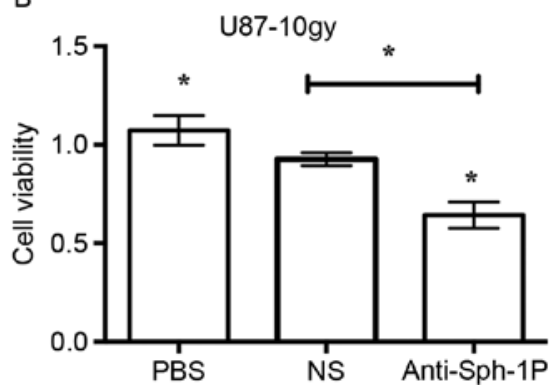

Figure 6. Neutralization of secreted ASAH1 and Sph-1P with Anti-ASAH1 or Anti-Sph-1P antibodies resulted in reduced cell growth. Cells were grown in 96-well plates with serum-free media over a period of $48 \mathrm{~h}$, after which MTT assays were performed to measure cell growth. U87-10gy cells were treated with $3 \mu \mathrm{g}$ of either anti-ASAH1 (A) or humanized anti-Sph-1P (LT1009) antibodies (B). PBS, and/or $3 \mu \mathrm{g}$ of non-specific IgG (NS), either control mouse or humanized IgG, were used as negative controls. Results shown are mean \pm SD of three independent experiments. ${ }^{*}<<0.05$, compared with PBS and NS (non-specific $\mathrm{IgG}$ ) groups.

A

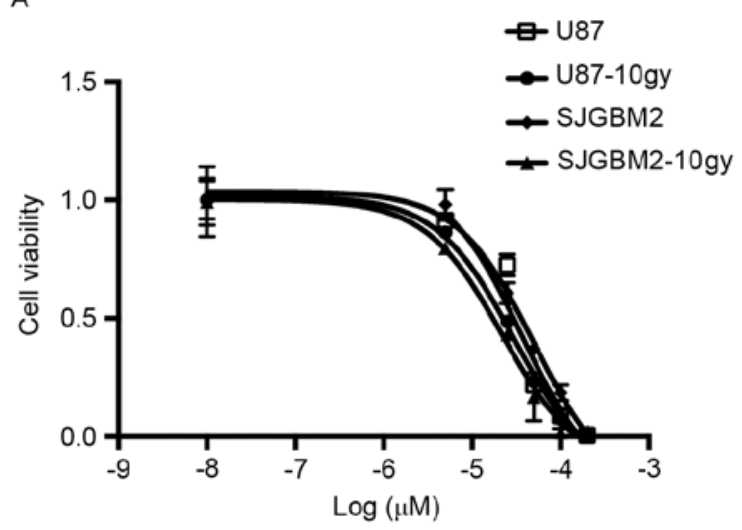

B

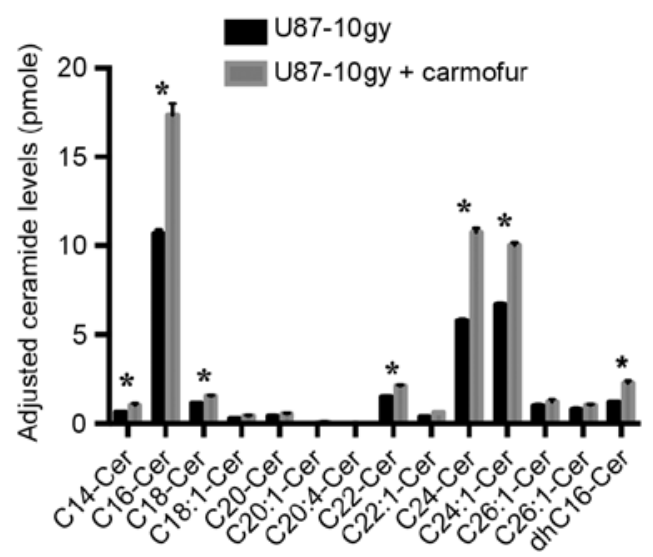

Figure 7. U87-10gy and SJGBM2-10gy cells are sensitive to carmofur and inhibition of ASAH1 with carmofur leads to increased levels of ASAH1 substrate ceramides. (A) Cells treated with carmofur and their $\mathrm{IC}_{50}$ values of 37, 50, 28 and $21 \mu \mathrm{M}$ for U87, SJGBM2, U87-10gy and SJGBM2-10gy, respectively, were determined utilizing MTT assays. (B) Intracellular ceramide levels were measured from untreated U87-10gy cells and U87-10gy cells treated with 50 $\mu \mathrm{M}$ carmofur for $1 \mathrm{~h}$. Concentrations were normalized and adjusted based on the phosphate level. $\mathrm{C}(\mathrm{N})$, ceramide species with long-N-acyl-chain; dhC, dihydroceramides. Results shown are mean \pm SD of three independent experiments. ${ }^{*} \mathrm{P}<0.05$.

in reduced cell growth. ASAH1, as shown in this study, can be secreted into the culture media. Similarly, Sph-1P can, as reported by others, be secreted into the media as well (21). Given their known roles in the promotion of cell growth (as shown in Fig. 5), we sought to determine whether neutralization of ASAH1 and Sph-1P with antibodies would lead to decreased cell growth. We treated U87-10gy with anti-ASAH1 and anti-Sph-1P antibodies for $48 \mathrm{~h}$ and measured cell growth patterns with MTT assays. Treatment of U87-10gy cells with $3 \mu \mathrm{g}$ of either anti-ASAH1 or anti-Sph-1P decreased cell growth by $\sim 50 \%$ (Fig. 6). The data imply that the neutralization of secreted ASAH1 or Sph-1P can prevent these molecules from promoting cell growth and proliferation.

Treatment of U87-10gy and SJGBM2-10gy cells with carmofur, an ASAH1 inhibitor, resulted in cell death and elevated levels of ceramides. Other researchers have demonstrated that an ASAH1 inhibitor, such as carmofur, can effectively target cancers (22). Previous data suggested that carmofur inhibits ASAH1 activity and elevates tissue ceramide levels, which in turn induces apoptosis (22). To test whether ASAH1 inhibition contributes to cell death, we evaluated the effects of carmofur on U87, SJGBM2, U87-10gy, and SJGBM-10gy cells. When exposed for $12 \mathrm{~h}$ to carmofur, all cell lines showed markedly increased cell death relative to control cells subjected to the same treatment. We observed a median inhibitory concentration $\left(\mathrm{IC}_{50}\right)$ of $37,50,28$ and $21 \mu \mathrm{M}$ for U87, SJGBM2, U87-10gy and SJGBM2-10gy, respectively (Fig. 7). Importantly, targeting U87-10gy cells with carmofur was accompanied by marked intracellular accumulation of various ceramide species compared to control cells (Fig. 7). This suggested that ASAH1 inhibition by carmofur contributes to cytotoxicity.

Irradiated GBMs exhibit significantly greater ASAH1 staining than non-irradiated GBMs. To evaluate the difference in ASAH1 IHC staining between irradiated and non-irradiated GBMs, we performed ASAH1 IHC on 6 irradiated and 5 nonirradiated GBMs. We scored the level of staining using the Allred scoring system, in which a higher score suggests more staining (18). Utilizing this system, we obtained the score of 5 for non-irradiated vs. 7 for irradiated GBMs, with a 


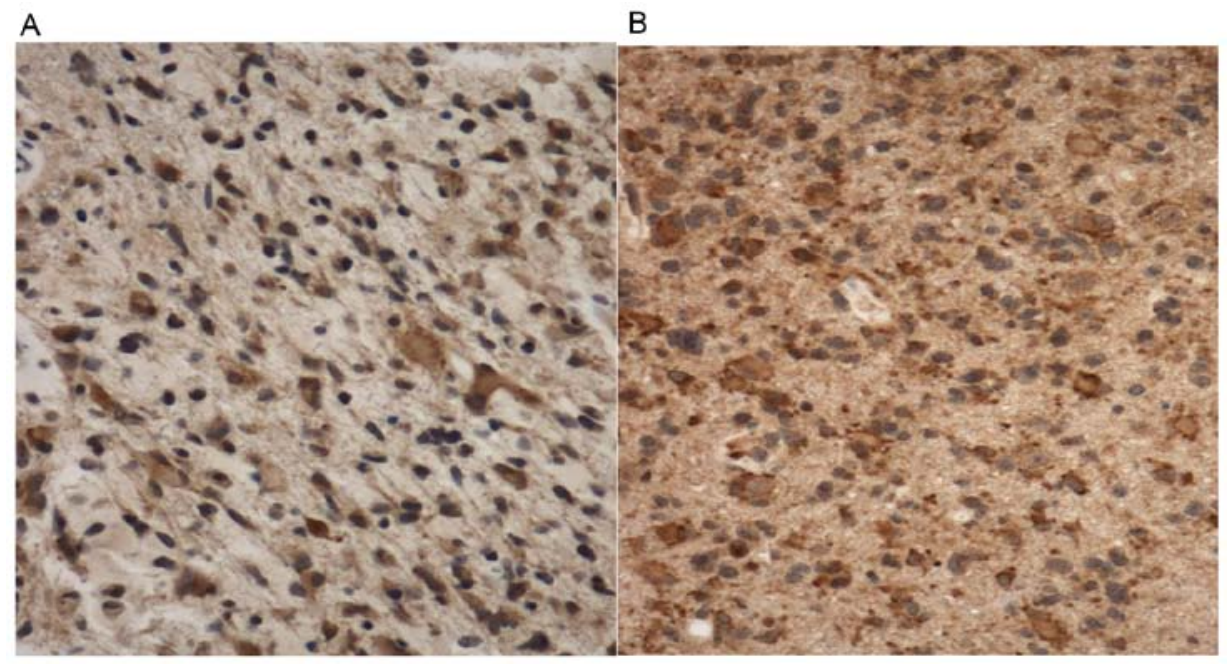

Figure 8. Irradiated GBMs exhibit significantly greater ASAH1 staining than de novo or non-irradiated GBMs. Representatives of ASAH1 IHC staining of non-irradiated (A) and irradiated GBMs (B) are shown. Similar to the irradiated U87 cells, secretion of ASAH1 may contribute to the higher extracellular staining of irradiated GBM.

Table I. Irradiated GBMs exhibit a statistically significantly higher Alfred ASAH1 staining score than non-irradiated GBMs.

\begin{tabular}{lccc}
\hline Characteristics & $\begin{array}{c}\text { Non-irradiated } \\
\text { GBM }\end{array}$ & $\begin{array}{c}\text { Irradiated } \\
\text { GBM }\end{array}$ & P-value \\
\hline Male & 3 & 3 & \\
Female & 2 & 3 & \\
Mean age (years) & $63 \pm 7$ & $57 \pm 6$ & \\
Alfred median IHC score & 5 & 7 & 0.036
\end{tabular}

Five non-irradiated and 6 irradiated GBMs were stained with the ASAH1 antibody and their stainings were scored using the Alfred scoring system taking into account both the proportion of positively stained tumor cells and the staining intensity. Non-irradiated and irradiated GBMs had an Alfred median score of 5 and 7, respectively with P-value of 0.036 .

statistically significant p-value (Table I and Fig. 8). Similar to Fig. 4, irradiated GBMs also exhibited higher ASAH1 staining in the extracellular space, possibly due to the secretion of ASAH1 into the extracellular space by irradiated GBMs (Fig. 8).

\section{Discussion}

The current standard treatment protocol for GBM consists of surgery followed by radiation and chemotherapy $(9,23)$. Despite treatment, all GBMs will inevitably develop resistance and recur $(12,24,25)$. It was postulated that GBM cancer stem cells (CSCs) are the culprit that promotes radioresistance, as evidenced by CD133-carrying glioma cells that were increased in proportion following ionizing radiation (13). A recent study in prostate cancer suggested that upregulation of ASAH1 confers resistance to radiation by altering the sphingolipid metabolism pathway (14). Our work addresses whether ASAH1 plays a similar role in GBM.
Western blot analysis of GBM cell cultures that survived 10 Gy of radiation (U87-10gy and SJGBM-10gy) revealed that protein expression of ASAH1 is significantly increased, while ceramide levels correspondingly decreased, when compared to non-irradiated GBM cells (Figs. 1 and 2). It has been shown in prostate cancers that upregulation of ASAH1 (following radiation) is mediated by radiation-induced c-Jun/AP-1, transcription factors that have been implicated in the DNA-repair pathway (15). ASAH1 is the principal, rate-limiting enzyme that metabolizes ceramides into sphingosine $(3,26,27)$. As expected from this mechanism, ceramide levels were decreased in cells containing a high level of ASAH1, as shown in U87-10gy and SJGBM2-10gy cells. However, Mahdy et al reported that upregulation of ASAH1 in prostate cancer cells did not result in lower ceramide levels (14). This discrepancy can be due to the timing of the measurement following radiation. In their study, the sphingolipid analysis was performed within hours following radiation; on the other hand, in our study, the analysis was performed once survived cells grew to confluence, a process that took approximately one month. Following radiation, most cells died within one week; $<1 \%$ of cells survived ionizing radiation and grew to confluence after a month of culture. The data suggest that only cells that express a high level of ASAH1 could survive radiation. Since Mahdy et al performed sphingolipid analysis within hours following radiation, their results likely included cells that would not survive radiation long-term (those that contained lower levels of ASAH1 and higher levels of ceramides). With our study, the longer time interval selected out these cells (as they died within 1 week), where final analysis involved only cells that survived radiation.

To confirm the upregulation of ASAH1 and Sph-1P as a mechanism of radioresistance, we performed western blotting and IHC on GBM cell lines and patient GBM tissues. IHC staining of both U87 and U87-10gy cells with humanized anti-Sph-1P revealed increased levels of Sph-1P in irradiated U87-10gy (Fig. 3). Similar to the western blot data, ASAH1 IHC analysis of four different sets of data from the same patient (pre- and post-radiation GBM specimens) confirmed 
the upregulation of ASAH1 in post-radiation samples, ranging from 1.5- to 60-fold higher in staining intensity as assessed by ImageJ (Fig. 4). This finding was further supported by data showing a significantly lower Allred median ASAH1 staining score for non-irradiated GBMs in comparison to radiated GBM samples (Fig. 8). Consistent with previous data (13), we showed that irradiated GBMs also have a higher protein expression of CD133 than non-irradiated GBMs. Given the concomitant high expression level of ASAH1 in irradiated GBMs, this raises the possibility that $\mathrm{CD}_{133^{+}}$cells or CSCs are the cells that survive radiation and overexpress ASAH1, as shown in western blot and IHC studies (Fig. 4A). These results indicate that the U87-10gy cell line is a potential, clinicallyrelevant model to study recurrent GBMs, especially in studies that target the sphingolipid metabolism pathway.

ASAH1 was shown in this study to be secreted into the extracellular space (Figs. 4, 5, and 8), which is consistent with other reports that document secretion of Sph-1P into the extracellular space as well $(21,26,28,29)$. Consequently, cancer cells with increased secretion of ASAH1 and Sph-1P create a tumor microenvironment that favors cancer survival by virtue of the ASAH1 and Sph-1P known tumor-promoting functions $(21,27,29-31)$. In support of this microenvironment theory, we demonstrated that media from SJGBM2-10gy cells, which secreted a high amount of ASAH1, promoted $50 \%$ more cell growth than media from SJGBM2 cells that contained a lower amount of secreted ASAH1 (Fig. 5). In addition, staining of irradiated GBMs also demonstrated significant ASAH1 staining in the extracellular space, suggesting that irradiated GBMs also secrete ASAH1 into the extracellular space (Figs. 4 and 8). The presence of tumor promoters ASAH1 and Sph-1P outside the intracellular space provides a unique opportunity to target these molecules with antibodies. Employing this strategy, we found that treatment of U87-10gy cells with anti-ASAH1 antibody reduced cell growth by $50 \%$ (Fig. 6). A similar $50 \%$ reduction in cell growth was observed in U87-10gy treated with the humanized anti-Sph-1P antibody (Fig. 6). This reduction in cell growth is likely attributed to the ability of antibodies to disrupt the roles that ASAH1 and Sph-1 have in the promotion of cell growth and survival (3,21,28,29,31-33). The benefit of an anti-ASAH1 antibody was clearly displayed in a serum autoantibody profiling study of patients with melanoma (34).

This study found that melanoma patients who developed auto anti-ASAH1 antibody were protected from lymph node metastasis. The study even suggested that upregulation of auto anti-ASAH1 antibody may play an important preventative role in melanoma metastasis, and the loss of this antibody may result in melanoma progression (34). With regards to the benefit of anti-Sph-1P antibody, multiple animal studies have shown that anti-Sph-1P antibody can neutralize the ability of Sph-1P to induce cell proliferation, promote angiogenesis, and protect tumor cells from apoptosis in several tumor cell lines $(16,35)$. In addition, Sph-1P has also been shown to be an important player in promoting malignancy in GBMs. Results from a previous study indicated that GBM malignancy is associated with an increased drive of the pathway that converts ceramide to Sph-1P (36). To explore the potential therapeutic benefit of inhibiting ASAH1 activity, we examined the effect of carmofur on cell survival. Carmofur decreased U87, SJGBM2,
U87-10gy and SJGBM-10gy cell viability with $\mathrm{IC}_{50}$ values of $37,50,28$, and $21 \mu \mathrm{M}$, respectively (Fig. 7). Irradiated cells are more sensitive to carmofur than their non-irradiated counterparts, possibly due to higher expression levels of ASAH1 in the former (Fig. 7). Treatment of U87-10gy cells with carmofur increased ceramide levels (Fig. 7).

In conclusion, GBM is a highly malignant tumor. Radiation is a mainstay treatment option. Despite aggressive management, the pathology inevitably recurs and/or progresses. This study provides an explanation as to why radiation treatments of GBM often have limited success, mainly due to the upregulation of ASAH1 and Sph-1P, leading to resistance to radiation. This study identifies ASAH1 and Sph-1P as excellent drug targets that can be taken advantage of to improve outcome. Inhibition of ASAH1 and Sph-1P, with antibodies, small molecule drugs (carmofur), or a combination of both, could represent an innovative clinical approach for treating GBMs, especially for ASAH1-overexpressed recurrent GBMs.

\section{Acknowledgements}

This study was funded by the Musella Foundation Grant and Department of Neurosurgery Larson Endowment Grant.

\section{References}

1. Mao C and Obeid LM: Ceramidases: Regulators of cellular responses mediated by ceramide, sphingosine, and sphingosine1-phosphate. Biochim Biophys Acta 1781: 424-434, 2008.

2. Vethakanraj HS, Babu TA, Sudarsanan GB, Duraisamy PK and Ashok Kumar S: Targeting ceramide metabolic pathway induces apoptosis in human breast cancer cell lines. Biochem Biophys Res Commun 464: 833-839, 2015.

3. Liu X, Elojeimy S, Turner LS, Mahdy AE, Zeidan YH, Bielawska A, Bielawski J, Dong JY, El-Zawahry AM, Guo GW, et al: Acid ceramidase inhibition: a novel target for cancer therapy. Front Biosci 13: 2293-2298, 2008.

4. Seelan RS, Qian C, Yokomizo A, Bostwick DG, Smith DI and Liu W: Human acid ceramidase is overexpressed but not mutated in prostate cancer. Genes Chromosomes Cancer 29: 137-146, 2000.

5. Roh JL, Park JY, Kim EH and Jang HJ: Targeting acid ceramidase sensitises head and neck cancer to cisplatin. Eur J Cancer 52: 163-172, 2016.

6. Realini N, Palese F, Pizzirani D, Pontis S, Basit A, Bach A, Ganesan A and Piomelli D: Acid ceramidase in melanoma: Expression, localization and effects of pharmacological inhibition. J Biol Chem 291: 2422-2434, 2016.

7. Morales A, París R, Villanueva A, Llacuna L, García-Ruiz C and Fernández-Checa JC: Pharmacological inhibition or small interfering RNA targeting acid ceramidase sensitizes hepatoma cells to chemotherapy and reduces tumor growth in vivo. Oncogene 26: 905-916, 2007

8. Morad SA, Messner MC, Levin JC, Abdelmageed N, Park H, Merrill AH Jr and Cabot MC: Potential role of acid ceramidase in conversion of cytostatic to cytotoxic end-point in pancreatic cancer cells. Cancer Chemother Pharmacol 71: 635-645, 2013.

9. Stupp R, Mason WP, van den Bent MJ, Weller M, Fisher B, Taphoorn MJ, Belanger K, Brandes AA, Marosi C, Bogdahn U, et al; European Organisation for Research and Treatment of Cancer Brain Tumor and Radiotherapy Groups; National Cancer Institute of Canada Clinical Trials Group: Radiotherapy plus concomitant and adjuvant temozolomide for glioblastoma. $\mathrm{N}$ Engl J Med 352: 987-996, 2005.

10. Iwamoto FM, Abrey LE, Beal K, Gutin PH, Rosenblum MK, Reuter VE, DeAngelis LM and Lassman AB: Patterns of relapse and prognosis after bevacizumab failure in recurrent glioblastoma. Neurology 73: 1200-1206, 2009.

11. Wong ET, Hess KR, Gleason MJ, Jaeckle KA, Kyritsis AP, Prados MD, Levin VA and Yung WK: Outcomes and prognostic factors in recurrent glioma patients enrolled onto phase II clinical trials. J Clin Oncol 17: 2572-2578, 1999. 
12. Lamborn KR, Yung WK, Chang SM, Wen PY, Cloughesy TF, DeAngelis LM, Robins HI, Lieberman FS, Fine HA, Fink KL, et al; North American Brain Tumor Consortium: Progression-free survival: An important end point in evaluating therapy for recurrent high-grade gliomas. Neuro Oncol 10: 162-170, 2008.

13. Bao S, Wu Q, McLendon RE, Hao Y, Shi Q, Hjelmeland AB, Dewhirst MW, Bigner DD and Rich JN: Glioma stem cells promote radioresistance by preferential activation of the DNA damage response. Nature 444: 756-760, 2006.

14. Mahdy AE, Cheng JC, Li J, Elojeimy S, Meacham WD, Turner LS, Bai A, Gault CR, McPherson AS, Garcia N, et al: Acid ceramidase upregulation in prostate cancer cells confers resistance to radiation: $\mathrm{AC}$ inhibition, a potential radiosensitizer. Mol Ther 17: 430-438, 2009.

15. Cheng JC, Bai A, Beckham TH, Marrison ST, Yount CL, Young K, Lu P, Bartlett AM, Wu BX, Keane BJ, et al: Radiationinduced acid ceramidase confers prostate cancer resistance and tumor relapse. J Clin Invest 123: 4344-4358, 2013.

16. Visentin B, Vekich JA, Sibbald BJ, Cavalli AL, Moreno KM, Matteo RG, Garland WA, Lu Y, Yu S, Hall HS, et al: Validation of an anti-sphingosine-1-phosphate antibody as a potential therapeutic in reducing growth, invasion, and angiogenesis in multiple tumor lineages. Cancer Cell 9: 225-238, 2006.

17. O'Brien N, Jones ST, Williams DG, Cunningham HB, Moreno K, Visentin B, Gentile A, Vekich J, Shestowsky W, Hiraiwa M, et al: Production and characterization of monoclonal anti-sphingosine1-phosphate antibodies. J Lipid Res 50: 2245-2257, 2009.

18. Allred DC, Harvey JM, Berardo M and Clark GM: Prognostic and predictive factors in breast cancer by immunohistochemical analysis. Mod Pathol 11: 155-168, 1998.

19. Bielawski J, Szulc ZM, Hannun YA and Bielawska A Simultaneous quantitative analysis of bioactive sphingolipids by high-performance liquid chromatography-tandem mass spectrometry. Methods 39: 82-91, 2006.

20. Visentin B, Reynolds G and Sabbadini R: Immunohistochemical detection of sphingosine-1-phosphate and sphingosine kinase-1 in human tissue samples. Methods Mol Biol 874: 55-67, 2012.

21. Ogretmen B and Hannun YA: Biologically active sphingolipids in cancer pathogenesis and treatment. Nat Rev Cancer 4: 604-616, 2004.

22. Realini N, Solorzano C, Pagliuca C, Pizzirani D, Armirotti A, Luciani R, Costi MP, Bandiera T and Piomelli D: Discovery of highly potent acid ceramidase inhibitors with in vitro tumor chemosensitizing activity. Sci Rep 3: 1035, 2013

23. Lacroix M, Abi-Said D, Fourney DR, Gokaslan ZL, Shi W, DeMonte F, Lang FF, McCutcheon IE, Hassenbusch SJ, Holland $\mathrm{E}$, et al: A multivariate analysis of 416 patients with glioblastoma multiforme: Prognosis, extent of resection, and survival. J Neurosurg 95: 190-198, 2001.
24. Ballman KV, Buckner JC, Brown PD, Giannini C, Flynn PJ, LaPlant BR and Jaeckle KA: The relationship between six-month progression-free survival and 12-month overall survival end points for phase II trials in patients with glioblastoma multiforme. Neuro-oncol 9: 29-38, 2007.

25. Hou LC, Veeravagu A, Hsu AR and Tse VC: Recurrent glioblastoma multiforme: A review of natural history and management options. Neurosurg Focus 20: E5, 2006.

26. Hannun YA and Obeid LM: Principles of bioactive lipid signalling: Lessons from sphingolipids. Nat Rev Mol Cell Biol 9: $139-150,2008$

27. Park JH and Schuchman EH: Acid ceramidase and human disease. Biochim Biophys Acta 1758: 2133-2138, 2006.

28. Ségui B, Andrieu-Abadie N, Jaffrézou JP, Benoist H and Levade T: Sphingolipids as modulators of cancer cell death: Potential therapeutic targets. Biochim Biophys Acta 1758: 2104-2120, 2006

29. Young N and Van Brocklyn JR: Roles of sphingosine-1-phosphate (S1P) receptors in malignant behavior of glioma cells. Differential effects of S1P2 on cell migration and invasiveness. Exp Cell Res 313: 1615-1627, 2007.

30. Johnson IR, Parkinson-Lawrence EJ, Butler LM and Brooks DA: Prostate cell lines as models for biomarker discovery: Performance of current markers and the search for new biomarkers. Prostate 74: 547-560, 2014.

31. Young N, Pearl DK and Van Brocklyn JR: Sphingosine-1phosphate regulates glioblastoma cell invasiveness through the urokinase plasminogen activator system and CCN1/Cyr61. Mol Cancer Res 7: 23-32, 2009.

32. Pettus BJ, Chalfant CE and Hannun YA: Ceramide in apoptosis: An overview and current perspectives. Biochim Biophys Acta 1585: 114-125, 2002

33. Zeidan YH, Jenkins RW, Korman JB, Liu X, Obeid LM, Norris JS and Hannun YA: Molecular targeting of acid ceramidase: Implications to cancer therapy. Curr Drug Targets 9: 653-661, 2008.

34. Liu Y, He J, Xie X, Su G, Teitz-Tennenbaum S, Sabel MS and Lubman DM: Serum autoantibody profiling using a natural glycoprotein microarray for the prognosis of early melanoma. J Proteome Res 9: 6044-6051,2010.

35. Zhang L, Wang X, Bullock AJ, Callea M, Shah H, Song J, Moreno K, Visentin B, Deutschman D, Alsop DC, et al: Anti-S1P antibody as a novel therapeutic strategy for VEGFR TKI-resistant renal cancer. Clin Cancer Res 21: 1925-1934, 2015.

36. Abuhusain HJ, Matin A, Qiao Q, Shen H, Kain N, Day BW, Stringer BW, Daniels B, Laaksonen MA, Teo C, et al: A metabolic shift favoring sphingosine 1-phosphate at the expense of ceramide controls glioblastoma angiogenesis. J Biol Chem 288: 37355-37364, 2013. 